\title{
The Accuracy of Group Beta Streptococcus Rectovaginal Cultures at 35 to 37 Weeks of Gestation in Predicting Colonization Intrapartum
}

\author{
Farrah N. Hussain, MD ${ }^{1}$ Zainab Al-Ibraheemi, MD ${ }^{1}$ \\ Dyese Taylor, MD ${ }^{1}$ Melissa Chu Lam, MD ${ }^{1}$ \\ ${ }^{1}$ Division of Maternal Fetal Medicine, Department of Obstetrics and \\ Gynecology, Mount Sinai West, Icahn School of Medicine at Mount \\ Sinai, New York, New York \\ 2 Department of Population Health Science and Policy, Icahn School of \\ Medicine at Mount Sinai, New York, New York \\ ${ }^{3}$ Division of Maternal Fetal Medicine, Department of Obstetrics and \\ Gynecology, New York University Langone Health, New York, New York \\ Am J Perinatol Rep 2019;9:e302-e309.
}

Dawnette Lewis, MD, MPH ${ }^{1}$

\begin{abstract}
Address for correspondence Farrah N. Hussain, MD, Division of Maternal Fetal Medicine, Department of Obstetrics and Gynecology, Mount Sinai West, Icahn School of Medicine at Mount Sinai, 1000 10th Avenue, Suite 2A, New York, NY 10019

(e-mail: farrah.hussain@mountsinai.org).
\end{abstract}

\author{
Abstract \\ Keywords \\ - bacterial colonization \\ - bacteriuria \\ - chemoprophylaxis \\ - maternal infection \\ - neonatal infection \\ - obesity \\ - pregnancy \\ - group $\beta$ Streptococcus \\ - screening methods
}

Objective This study aims to investigate accuracy of group beta Streptococcus (GBS) rectovaginal cultures at 35 to 37 weeks in predicting intrapartum colonization.

Study Design Institutional review board (IRB) approved prospective cohort study of 302 women from October 2015 to May 2017. Patients had the following tests for GBS: first trimester urine culture, rectovaginal culture at 35 to 37 weeks, and intrapartum rectovaginal culture. Outcomes included accuracy of 35- to 37-week GBS rectovaginal culture in detecting results intrapartum, and accuracy of first trimester urine culture in comparison to intrapartum rectovaginal cultures.

Results There was sufficient evidence of agreement between results at 35 to 37 weeks with intrapartum cultures $(p=0.001)$. However, agreement was weak, 11 patients (3.7\%) were GBS positive intrapartum but negative at 35 to 37 weeks; and 33 patients (11\%) were initially GBS positive but were negative intrapartum. Sensitivity and specificity of the 35 - to 37 -week culture was $69 \%$ (95\% confidence interval [Cl]:54-84\%) and $87 \%$ (95\% Cl: $83-91 \%)$, respectively. There was also weak agreement between first trimester urine culture and intrapartum rectovaginal culture. Specificity for this assessment was $98 \%(95 \% \mathrm{Cl}: 97-100 \%)$ and was significantly different compared with antepartum GBS culture $(p<0.001)$. Accuracy between antepartum GBS rectovaginal culture and urine culture was similar ( 85 vs. $87 \%, p=0.47$ ).

Conclusion The 35- to 37-week GBS rectovaginal culture might be a poor predictor for intrapartum colonization.

Group beta Streptococcus (GBS) is a common colonizer of the maternal genital tract. This bacterium can be passed to the fetus during gestation or during passage through the birth canal. Infection can manifest as meningitis, pneumonia, sepsis, and can result in fetal or neonatal demise. ${ }^{1}$ Surviving infants can present with mental retardation, hearing, or

received

May 6, 2019

accepted after revision

June 28, 2019
DOI https://doi.org/

$10.1055 / \mathrm{s}-0039-1697655$. ISSN 2157-6998. vision loss, while maternal infection can result in chorioamnionitis and endometritis. ${ }^{2}$ Colonization with GBS as a leading cause of early neonatal morbidity and mortality in the United States emerged in the 1970s. In the 1980s, several clinical trials and observational studies demonstrated the effectiveness of intrapartum antibiotic chemoprophylaxis in
Copyright $\odot 2019$ by Thieme Medical Publishers, Inc., 333 Seventh Avenue, New York, NY 10001, USA. Tel: +1(212) 584-4662.
License terms

(요 (1) $\circledast$ 
decreasing the risk of women transmitting GBS to their newborns, thereby preventing early-onset neonatal disease. ${ }^{3-7}$ In 1996, initial guidelines by the American College of Obstetricians and Gynecologists (ACOG), the Centers for Disease Control (CDC) and the American Academy of Pediatrics (AAP) recommended intrapartum GBS prophylaxis. Subsequently, in 2002, these guidelines were revised to include universal culture-based screening of all pregnant women between 35 and 37 weeks of gestational age to identify necessary intrapartum prophylaxis. ${ }^{8}$ These guidelines and public health efforts coincided with a steep decline in the incidence of early-onset neonatal disease by $65 \%$ from 1.7 per 1,000 live births in 1993 to 0.6 per 1,000 live births in 1998 and 0.34 to 0.37 per 1,000 live births in 2002 to 2008 . Similarly, the incidence of invasive disease in pregnant women also declined by $80 \%$ since the early 1990 s, when implementation of intrapartum antibiotic prophylaxis to prevent GBS disease began., 2,8

Despite universal screening and intrapartum prophylaxis efforts, GBS disease still remains as a leading cause of maternal uterine infection and morbidity and mortality among newborns. ${ }^{3}$ The evidence on the accuracy of GBS rectovaginal culture at 35 to 37 weeks is very limited. The objective of this study was to investigate the accuracy of GBS rectovaginal cultures at 35 to 37 weeks (antepartum) in predicting GBS colonization intrapartum.

\section{Materials and Methods}

This is an institutional review board (IRB) approved prospective cohort study (IRB: 14-0157) of 302 patients admitted to labor and delivery at term (37 weeks or greater) from October 2015 to May 2017. All patients had following two screening tests for GBS colonization as part of their prenatal care: a general urine culture during the first trimester and a rectovaginal culture performed at 35 to 37 weeks of gestational age. In addition, after obtaining informed consent, a rectovaginal culture was obtained intrapartum prior to administration of any intrapartum antibiotics. Patients were excluded if they were preterm ( $<37$ weeks' gestation), presented for elective Cesarean delivery, had unknown GBS status, presented with chorioamnionitis and received antibiotics prior to study entry, had any autoimmune disorders, if they were HIV positive, or if they were unable to provide written consent to participate in the study. Patient demographics, gestational age at admission, maternal complications (chorioamnionitis, preeclampsia, postpartum hemorrhage, gestational diabetes, and endometritis), neonatal complications (meconium staining, shoulder dystocia, small for gestational age [SGA], and other complications), and delivery outcomes were collected from the patient and abstracted from the chart.

The collection of all rectovaginal cultures were performed by trained resident and attending physicians in the outpatient and inpatient settings. The rectovaginal cultures were collected using a standardized procedure as outlined by the ACOG and the 2010 CDC guidelines. ${ }^{8,9}$ In particular, a single swab was inserted into the vaginal introitus, followed by the rectum, and into the anal sphincter. All cultures were collected using BBL
CultureSwab Plus (Becton, Dickinson and Company, Sparks, MD) with amies gel and incubated on Lim broth for 24 hours. At 24 hours, the broth was subcultured to blood agar (BAP) and Columbia naladixic agar (CAN) plates. Identification of GBS also included Gram smear and catalase testing.

The primary outcome was to evaluate the accuracy of the 35- to 37-week of GBS rectovaginal culture in detecting the results of the intrapartum GBS rectovaginal culture. The secondary outcomes assessed the accuracy of first trimester urine culture in comparison to the results obtained at the intrapartum GBS rectovaginal culture. We explored the comparison between the two diagnostic results in their sensitivity, specificity, positive predictive value (PPV), negative predictive value (NPV), and accuracy. Additional exploratory analyses included demographic, maternal, and neonatal outcomes are provided descriptively and comparatively, between GBS colonization results.

Continuous variables were reported as mean and standard deviation (SD) or median and interquartile range (IQR). Categorical variables on demographics, maternal, and neonatal outcomes were reported as frequencies and column proportions. Comparisons in GBS colonization results were performed using Student's $t$-test or Wilcoxon's test for continuous variables, as appropriate or Chi-square test or Fisher's exact test for categorical variables, as appropriate. To assess the agreement between the GBS rectovaginal culture at 35 to 37 weeks and first trimester urine culture with the intrapartum GBS rectovaginal culture, we reported Cohen's kappa (k) coefficient with a $p$-value computed under the null hypothesis that agreement is by chance alone. We defined a kappa coefficient less than 0.60 as weak, 0.60 to 0.80 as moderate, and above 0.80 as strong agreement between the two diagnostic tests. Measures of agreement (i.e., sensitivity, specificity, PPV, NPV, and accuracy) to intrapartum GBS rectovaginal culture were reported for 35 to 37 weeks GBS rectovaginal culture and first trimester urine culture with respective 95\% confidence intervals (CIs). Comparisons in the measures of agreement between 35 and 37 weeks of GBS rectovaginal cultures and urine cultures were performed using a Chi-square test.

Although prior studies have compared antepartum and intrapartum cultures, their results have been varied. ${ }^{10-12}$ Therefore, it was difficult to calculate a sample size based on a proportional difference between the 35 and 37 weeks of culture and the intrapartum culture. We therefore based our sample size calculation on data showing that the 35- to 37week GBS rectovaginal culture relative to rapid tests have a sensitivity of $42.3 \%$ (95\% CI: 36-49\%) and specificity of $100 \%$ (95\% CI: 99-100\%). ${ }^{13}$ The prevalence of colonization of GBS in pregnant women has been estimated to be 10 to $30 \%{ }^{8}$ Assuming a sample size of 300 patients and prevalence of colonization to be $10 \%$ provided an NPV estimate of $94.1 \%$ with a $95 \%$ CI of 92.2 to $95.6 \%$ in precision.

\section{Results}

After excluding three patients who did not have a 35- to 37week GBS rectovaginal culture, a total of 299 patients were included in the analysis assessing agreement between 35 and 
Table 1 Comparison of first trimester urine culture, GBS rectovaginal culture at 35 to 37 weeks and intrapartum GBS rectovaginal culture

\begin{tabular}{|c|c|c|c|c|}
\hline \multirow[t]{2}{*}{ GBS culture at $35-37$ weeks } & \multicolumn{2}{|c|}{$\begin{array}{l}\text { Intrapartum GBS Culture } \\
n=299(\%)\end{array}$} & \multirow[t]{2}{*}{ Kappa $(95 \% \mathrm{Cl})$} & \multirow[t]{2}{*}{$p$-Value } \\
\hline & Negative & Positive & & \\
\hline Negative & $230(76.9)$ & $11(3.7)$ & \multirow[t]{2}{*}{$0.45(0.32-0.58)$} & \multirow[t]{2}{*}{$<0.001$} \\
\hline Positive & $33(11)$ & $25(8.4)$ & & \\
\hline \multirow[t]{2}{*}{ First trimester urine culture } & \multicolumn{2}{|c|}{$\begin{array}{l}\text { Intrapartum GBS culture } \\
n=284(\%)\end{array}$} & & \\
\hline & Negative & Positive & & \\
\hline Negative & $245(86.3)$ & $32(11.3)$ & \multirow[t]{2}{*}{$0.11(-0.03$ to 0.24$)$} & \multirow[t]{2}{*}{0.01} \\
\hline Positive & $4(1.4)$ & $3(1.1)$ & & \\
\hline \multirow[t]{2}{*}{ First trimester urine culture } & \multicolumn{2}{|c|}{$\begin{array}{l}\text { GBS culture at } 35-37 \text { weeks } \\
n=282(\%)\end{array}$} & & \\
\hline & Negative & Positive & & \\
\hline Negative & $228(80.9)$ & $49(17.4)$ & \multirow[t]{2}{*}{$0.08(-0.02$ to 0.17$)$} & \multirow[t]{2}{*}{0.02} \\
\hline Positive & $2(0.7)$ & $3(1.1)$ & & \\
\hline
\end{tabular}

Abbreviations: $\mathrm{Cl}$, confidence interval; GBS, group beta Streptococcus.

37 weeks of GBS rectovaginal culture with their intrapartum GBS rectovaginal cultures. Eleven (3.7\%) patients who had a negative GBS rectovaginal culture at 35 to 37 weeks tested positive for GBS intrapartum. Thirty-three (11\%) patients who had a positive GBS rectovaginal culture at 35 to 37 weeks tested negative for GBS intrapartum. Cohen's kappa test of agreement was performed between the test results and found that there was sufficient evidence of an agreement in the test results at 35 to 37 weeks and intrapartum ( - Table 1). However, the level of agreement was weak $(k=0.45 ; 95 \% \mathrm{Cl}$ : $0.32-0.58)$. The sensitivity and specificity of the 35- to 37-week GBS rectovaginal culture with the intrapartum GBS rectovaginal culture were 69\% (95\% CI: 54-84) and 87\% (95\% CI: 83-91), respectively. The positive and negative predictive values of the antepartum GBS rectovaginal culture were $43 \%$ (95\% CI: 30-56) and 95\% (95\% CI: 93-98), respectively, with an accuracy of $85 \%$ (95\% CI: 81-89; - Table 2).

Baseline demographics and clinical characteristics of women who had negative results in both the 35 to 37 weeks and intrapartum GBS rectovaginal cultures were compared with those who were positive at the time of delivery
(-Table 3). There was no significant difference between the two groups with respect to demographic data and maternal and neonatal outcome except for a higher pregravid body mass index (BMI; $24.62 \pm 5.32$ vs. $31.34 \pm 6.55$, $p<0.001$ ) and parity (0 [IQR: $0-1$ ] vs. 1 [IQR: 0-3], $p=0.002$ ) in patients who were newly GBS positive at delivery.

Baseline demographics and clinical characteristics of women who had positive GBS rectovaginal cultures at 35 to 37 weeks, including those who were false positives, were compared with those who were only detected positive intrapartum (-Table 4). Again, there was no significant difference between the two groups with respect to demographic data and maternal and neonatal outcome except for a higher pregravid BMI $(31.34 \pm 6.55$ vs. $24.38 \pm 5.17, p<0.001)$ and parity ( 0 vs. $1, p=0.008$ ) in patients who were newly GBS positive at delivery.

The first trimester urine culture was compared with GBS rectovaginal culture at 35 to 37 weeks (-Table $\mathbf{1}$ ). Twenty patients either did not complete a urine culture or a GBS rectovaginal culture at 35 to 37 weeks. These patients were

Table 2 Measures of agreement to intrapartum GBS rectovaginal culture for 35 to 37 weeks' GBS rectovaginal culture and first trimester urine culture

\begin{tabular}{|c|c|c|c|}
\hline & $\begin{array}{l}\text { GBS rectovaginal culture at } \\
35-37 \text { weeks with intrapartum } \\
\text { GBS rectovaginal culture in } \%(95 \% \mathrm{Cl})\end{array}$ & $\begin{array}{l}\text { First trimester urine culture } \\
\text { with intrapartum GBS } \\
\text { culture in } \%(95 \% \mathrm{Cl})\end{array}$ & $p$-Value \\
\hline Sensitivity & $69(54-84)$ & $9(0-18)$ & $<0.001$ \\
\hline Specificity & $87(83-91)$ & $98(97-100)$ & $<0.001$ \\
\hline Positive predictive value & $43(30-56)$ & $43(6-80)$ & $>0.99$ \\
\hline Negative predictive value & $95(93-98)$ & $88(85-92)$ & 0.004 \\
\hline Accuracy & $85(81-89)$ & $87(83-91)$ & 0.47 \\
\hline
\end{tabular}

Abbreviations: $\mathrm{Cl}$, confidence interval; GBS, group beta Streptococcus. 
Table 3 Demographics and clinical characteristics of patients with negative GBS rectovaginal culture at 35 to 37 weeks and intrapartum compared with patients who had a newly positive GBS rectovaginal culture at delivery

\begin{tabular}{|c|c|c|c|}
\hline Demographics and clinical characteristics & $\begin{array}{l}\text { Negative GBS rectovaginal } \\
\text { culture at } 35-37 \text { weeks } \\
\text { and intrapartum } \\
n=230(\%)\end{array}$ & $\begin{array}{l}\text { Newly positive GBS } \\
\text { rectovaginal culture } \\
\text { at delivery } \\
n=11(\%)\end{array}$ & $p$-Value \\
\hline Age $(y)$ & $32.37 \pm 5.35$ & $32.27 \pm 5.80$ & 0.96 \\
\hline \multicolumn{3}{|l|}{ Race } & \multirow[t]{6}{*}{0.35} \\
\hline Asian & $37(16.1)$ & 0 & \\
\hline Black & $28(12.2)$ & $3(27.3)$ & \\
\hline Hispanic & $31(13.5)$ & $2(18.2)$ & \\
\hline White & $112(48.7)$ & $5(45.5)$ & \\
\hline Other & $22(9.6)$ & $1(9.1)$ & \\
\hline Pregravid BMI $\left(\mathrm{kg} / \mathrm{m}^{2}\right)$ & $24.62 \pm 5.32$ & $31.34 \pm 6.55$ & $<0.001$ \\
\hline Gravidity & $2(1-3)$ & $2(1-5)$ & 0.06 \\
\hline Parity & $0(0-1)$ & $1(0-3)$ & 0.002 \\
\hline Gestational age at admission (wk) & $39.54 \pm 1.12$ & $39.34 \pm 1.35$ & 0.57 \\
\hline Patients receiving Medicaid & $52(22.6)$ & $4(36.4)$ & 0.29 \\
\hline \multicolumn{3}{|l|}{ Mode of delivery } & \multirow[t]{4}{*}{$>0.99$} \\
\hline Vaginal & $150(65.2)$ & $8(72.7)$ & \\
\hline Cesarean & $44(19.1)$ & $2(18.2)$ & \\
\hline Operative vaginal & $36(15.7)$ & $1(9.1)$ & \\
\hline \multicolumn{3}{|l|}{ 1-minute Apgar's score } & \multirow[t]{3}{*}{$>0.99$} \\
\hline$<7$ & $17(7.4)$ & 0 & \\
\hline$\geq 7$ & $213(92.6)$ & $11(100)$ & \\
\hline \multicolumn{3}{|l|}{ 5-minute Apgar's score } & \multirow[t]{3}{*}{$>0.99$} \\
\hline$<7$ & $2(0.9)$ & 0 & \\
\hline$\geq 7$ & $228(99.1)$ & $11(100)$ & \\
\hline NICU admission & $5(2.2)$ & 0 & $>0.99$ \\
\hline \multicolumn{3}{|l|}{ Maternal complications } & \multirow[t]{8}{*}{0.31} \\
\hline None & $144(62.6)$ & $7(63.6)$ & \\
\hline Chorioamnionitis & $31(13.5)$ & 0 & \\
\hline Preeclampsia/gestational hypertension & $28(12.2)$ & $2(18.2)$ & \\
\hline Retained placenta/postpartum hemorrhage & $10(4.4)$ & 0 & \\
\hline Gestational diabetes mellitus & $12(5.2)$ & $1(9.1)$ & \\
\hline Endometritis & 0 & 0 & \\
\hline Other & $5(2.2)$ & $1(9.1)$ & \\
\hline \multicolumn{3}{|l|}{ Neonatal complications } & \multirow[t]{6}{*}{0.13} \\
\hline None & $186(80.9)$ & $8(72.7)$ & \\
\hline Light/moderate/thick meconium staining & $33(14.4)$ & $1(9.1)$ & \\
\hline Shoulder dystocia & $4(1.7)$ & 0 & \\
\hline Small for gestational age & $5(2.2)$ & $2(18.2)$ & \\
\hline Other & $2(0.9)$ & 0 & \\
\hline
\end{tabular}

Abbreviations: BMI, body mass index; GBS, group beta Streptococcus; NICU, neonatal intensive care unit.

Note: Continuous variables are reported as mean \pm standard deviation or median (interquartile range). Categorical variables are reported as frequency and column proportions. 
Table 4 Demographics and clinical characteristics of patients with a positive GBS rectovaginal culture at 35 to 37 weeks compared with patients who had a newly positive GBS rectovaginal culture at delivery

\begin{tabular}{|c|c|c|c|}
\hline Demographics and clinical characteristics & $\begin{array}{l}\text { Positive at } 35-37 \text { weeks' } \\
\text { GBS rectovaginal culture } \\
n=58(\%)\end{array}$ & $\begin{array}{l}\text { Newly positive GBS } \\
\text { rectovaginal culture at delivery } \\
n=11(\%)\end{array}$ & $p$-Value \\
\hline Age (y) & $32.86 \pm 5.81$ & $32.27 \pm 5.80$ & 0.76 \\
\hline \multicolumn{3}{|l|}{ Race } & \multirow[t]{6}{*}{0.74} \\
\hline Asian & $5(8.6)$ & 0 & \\
\hline Black & $19(32.8)$ & $3(27.3)$ & \\
\hline Hispanic & $4(6.9)$ & $2(18.2)$ & \\
\hline White & $25(43.1)$ & $5(45.4)$ & \\
\hline Other & $5(8.6)$ & $1(9.1)$ & \\
\hline Pregravid BMI $\left(\mathrm{kg} / \mathrm{m}^{2}\right)$ & $24.38 \pm 5.17$ & $31.34 \pm 6.55$ & $<0.001$ \\
\hline Gravidity & $2(1-3)$ & $2(1-5)$ & 0.17 \\
\hline Parity & $0(0-1)$ & $1(0-3)$ & 0.008 \\
\hline Gestational age at admission (wk) & $40.04 \pm 1.04$ & $39.34 \pm 1.35$ & 0.05 \\
\hline Patients receiving Medicaid & $18(31.0)$ & $4(36.4)$ & 0.74 \\
\hline \multicolumn{3}{|l|}{ Mode of delivery } & \multirow[t]{4}{*}{$>0.99$} \\
\hline Vaginal & $36(62.1)$ & $8(72.7)$ & \\
\hline Cesarean & $16(27.6)$ & $2(18.2)$ & \\
\hline Operative vaginal & $6(10.3)$ & $1(9.1)$ & \\
\hline \multicolumn{3}{|l|}{ 1-minute Apgar's score } & \multirow[t]{3}{*}{$>0.99$} \\
\hline$<7$ & $4(6.9)$ & 0 & \\
\hline$\geq 7$ & $54(93.1)$ & $11(100)$ & \\
\hline \multicolumn{3}{|l|}{ 5-minute Apgar's score } & \multirow[t]{3}{*}{$\mathrm{N} / \mathrm{A}$} \\
\hline$<7$ & 0 & 0 & \\
\hline$\geq 7$ & $58(100)$ & $11(100)$ & \\
\hline NICU admission & $1(1.7)$ & 0 & $>0.99$ \\
\hline \multicolumn{3}{|l|}{ Maternal complications } & \multirow[t]{8}{*}{0.25} \\
\hline None & $37(63.8)$ & $7(63.6)$ & \\
\hline Chorioamnionitis & $11(19)$ & 0 & \\
\hline Preeclampsia/gestational hypertension & $5(8.6)$ & $2(18.2)$ & \\
\hline Retained placenta/postpartum hemorrhage & $1(1.7)$ & 0 & \\
\hline Gestational diabetes mellitus & $2(3.5)$ & $1(9.1)$ & \\
\hline Endometritis & $1(1.7)$ & 0 & \\
\hline Other & $1(1.7)$ & $1(9.1)$ & \\
\hline \multicolumn{3}{|l|}{ Neonatal complications } & \multirow[t]{6}{*}{0.42} \\
\hline None & $42(73.7)$ & $8(72.7)$ & \\
\hline Light/moderate/thick meconium staining & $11(19.3)$ & $1(9.1)$ & \\
\hline Shoulder dystocia & $1(1.8)$ & 0 & \\
\hline Small for gestational age & $2(3.5)$ & $2(18.2)$ & \\
\hline Other & $1(1.8)$ & 0 & \\
\hline
\end{tabular}

Abbreviations: BMI, body mass index; GBS, group beta Streptococcus; N/A, not available; NICU, neonatal intensive care unit.

Note: Continuous variables are reported as mean \pm standard deviation or median (interquartile range). Categorical variables are reported as frequency and column proportions. 
excluded from the analysis assessing an agreement between first trimester urine culture and intrapartum GBS rectovaginal culture.

Forty-nine (17.4\%) patients had a negative GBS first trimester urine culture but were positive for GBS at the 35 - to $37-$-week rectovaginal culture. Two $(0.7 \%)$ patients had a positive GBS first trimester urine culture but were negative for rectovaginal GBS at 35 to 37 weeks. Cohen's kappa test of agreement was performed between the test results and found that there was a sufficient evidence of an agreement in the culture results at the first trimester and at 35 to 37 weeks $(p=0.02)$. However, the level of agreement was weak $(k=0.08 ; 95 \% \mathrm{CI}:-0.02$ to 0.17$)$. There were 49 patients $(17.4 \%)$ who had negative urine cultures were detected positive in their GBS cultures at 35 to 37 weeks and two patients $(0.7 \%)$ with false positive results ( - Table 1 ).

The first trimester urine culture was also compared with GBS rectovaginal culture intrapartum. Eighteen patients did not have a first trimester urine culture and were excluded from the analysis. Thirty-two (11.3\%) patients who had a negative first trimester urine culture were positive for GBS at delivery. There was sufficient evidence of an agreement between the two culture exams $(p=0.01)$, although this agreement was also weak $(k=0.11,95 \% \mathrm{CI}:-0.03$ to 0.24 ; - Table 1$)$. The sensitivity and specificity of the first trimester urine culture with intrapartum GBS rectovaginal culture were 9\% (95\% CI: 0-18) and 98\% (95\% CI: 97-100), respectively. The positive and negative predictive values of the first trimester culture with intrapartum GBS rectovaginal culture were 43\% (95\% CI: 30-56) and 88\% (95\% CI: 85-92), respectively, with an accuracy of $87 \%$ (95\% CI: 83-91; - Table 2).

\section{Clinical Implications}

Our study investigated the accuracy of antepartum GBS rectovaginal cultures at 35 to 37 weeks in predicting GBS colonization intrapartum. Although we found evidence of agreement between antepartum and intrapartum GBS culture results, the level of agreement was weak. The overall antepartum GBS culture accuracy is $85 \%$ with a sensitivity of $69 \%$ and specificity of $87 \%$. The $3.7 \%$ patients who had a negative GBS rectovaginal culture at 35 to 37 weeks tested positive for GBS intrapartum. Also, $11 \%$ of patients who had a positive GBS culture at 35 to 37 weeks tested negative for GBS intrapartum. The pregravid BMI and parity were higher in patients who were newly GBS positive at delivery. Similarly, there was evidence of agreement between first trimester urine culture with antepartum and intrapartum GBS cultures but both were weak. There were $17.4 \%$ of patients that were negative for GBS at the first trimester culture but were positive for GBS at the 35- to 37-week culture. Also, $11.3 \%$ patients who had a negative GBS culture at the first trimester urine culture were GBS positive at delivery.

It is well known that rectovaginal GBS colonization can change over the course of a pregnancy. ${ }^{10}$ In a prior prospective study, GBS culture performed between 23 and 26 weeks' gestation was not found to be a reliable predictor of earlyonset GBS disease. ${ }^{14}$ Another study performed by Boyer et al ${ }^{10}$ demonstrated an inverse relationship between the predictive value of GBS culture and the interval between prenatal sampling and delivery. This discordance may be explained by chronic intestinal GBS carriage, with intermittent seeding of the genital tract. ${ }^{15,16}$ Therefore, given the transient nature of rectovaginal GBS colonization, it is imperative to identify the ideal timing of specimen collection.

Recent studies examining the predictive value of antenatal GBS cultures are limited and tend to differ in their observations. A 1983 prospective study by Boyer et al ${ }^{10}$ screened 593 pregnant women at their prenatal visits and at delivery for rectovaginal GBS. A total of 393 patients had positive GBS cultures of whom 264 (67.2\%) remained positive at delivery. Out of 200 patients with negative antenatal screening, 17 (8.5\%) were positive for GBS when tested at delivery. Similar to our findings, the predictive value for a negative antenatal culture was 91.5 , and $67.2 \%$ for a positive prenatal culture, with a sensitivity of $70 \%$ and a specificity of $90.4 \%$. A 1996 study of 826 women also screened pregnant women at their 35- to 36-week prenatal care visit, and again at admission for delivery, but demonstrated greater accuracy of the antepartum results compared with intrapartum, revealing a sensitivity of $87 \%$, and specificity of $96 \%{ }^{11}$ Additionally, a 2010 systematic review examined the predictive value of antenatal GBS cultures in 8,898 patients in nine prospective and retrospective studies. Their findings included positive predictive values for antenatal cultures ranging from 43 to $100 \%$ (mean $69 \%$ ), and negative predictive values ranging from 80 to $100 \%$ (mean 94\%). In general, GBS cultures collected later in pregnancy had high-positive predictive values for colonization intrapartum. However, $6 \%$ of intrapartum GBS carriers remained undetected in antenatal cultures. Overall, these findings were limited due to varying study methods and incomplete follow-up information. $^{12}$

GBS is found in the urine of 2 to $7 \%$ of pregnant women and is a known marker for heavy maternal genital tract colonization. ${ }^{8}$ Similar to our results, prior studies have found that GBS bacteriuria in the first trimester are not necessarily indicative of GBS rectovaginal culture results at 35 to 37 weeks or at the time of delivery. ${ }^{17,18}$ These results emphasize the transient nature of colonization. However, as GBS bacteriuria places neonates at greater risk of early-onset disease, it is still included as an indication for intrapartum antibiotic prophylaxis. $^{8}$

In our baseline demographics, there was a higher pregravid BMI in patients who were found to be newly GBS positive at delivery. Although rectovaginal collection methods were standardized, there may have been inherent technical difficulties in obtaining samples from obese gravidas. Moreover, our study was not powered to detect differences in GBS colonization in obese versus nonobese patients. The association between maternal obesity and rectovaginal GBS colonization at term has been examined previously. A retrospective cohort study by Kleweis et al ${ }^{19}$ compared 4,492 obese women (BMI of $30 \mathrm{~kg} / \mathrm{m}^{2}$ or greater) to 3,219 nonobese women (BMI of less than $30 \mathrm{~kg} / \mathrm{m}^{2}$ ). Obese women were significantly more likely to be colonized with GBS at term than nonobese women (28.4 vs. $22.2 \%, p<0.001)$. Stapleton et $\mathrm{al}^{20}$ conducted a populationbased, case-control study of 40,459 cases of GBS colonization, 
and found a $20 \%$ increase in GBS colonization in women with a BMI greater than $30 \mathrm{~kg} / \mathrm{m}^{2}$, and a $45 \%$ increase in women with a BMI greater than $40 \mathrm{~kg} / \mathrm{m}^{2}$. This increase in GBS colonization may be the result of a shift in the gastrointestinal microbiome in obese patients, with an increase in Firmicutes (the phylum of GBS) and a decrease in Bacteroides species. ${ }^{19}$

As previously discussed, despite widespread adoption of 2002 recommendations for universal screening, GBS remains one of the most common infections in neonates. ${ }^{21}$ Moreover, given the transient nature of colonization, treatment of women based on 35 to 37 week rectovaginal cultures may lead to inappropriate chemoprophylaxis. A multistate, retrospective cohort study by Van Dyke et al ${ }^{22}$ examined 254 births in which the infant had GBS disease. Missed screening among patients who delivered at term accounted for 34 out of 254 (13.4\%) cases of neonatal GBS disease. In addition, 116 (61.4\%) term infants with GBS disease were born to women who had tested negative for GBS antepartum (false negative cases). This was the largest portion of GBS disease in term of infants in the study. ${ }^{22}$ Although we were neither powered for, nor did we observe any cases of infants with GBS disease, 3.7\% of patients who had a negative GBS culture at 35 to 37 weeks did test positive for GBS intrapartum. Combined with the results in the literature, one can ascertain the potential pitfalls of screening for a transient infection at 35 to 37 weeks.

\section{Research Implications}

Opportunity may exist to improve upon our current culturebased screening methods. Unlike standard culture methods which require at least 24 to 48 hours of incubation, real-time polymerase chain reaction (PCR) tests for rapid GBS screening can take as little as 99 minutes to complete which could make PCR an asset in the clinical setting. However, a drawback of PCR is lack of antibiotic susceptibility testing. Studies evaluating the sensitivity, specificity, and clinical utility of PCR are promising but limited. A Canadian study of 190 women comparing 35- to 37-week antepartum cultures to PCR demonstrated a sensitivity of 90.7\% (CI: 79.7-96.9) and specificity of $97.6 \%$ (95\% CI: 93.1-99.5), with $81 \%$ of results available more than 4 hours prior to delivery. ${ }^{23}$ Similarly, a 2018 meta-analysis of 15 studies indicated a sensitivity of 93.7\% (CI: 92.1-95.3) and specificity of 97.6\% (CI: 97.0-98.1). ${ }^{24}$ Given the strengths and pitfalls of both methods, further study, including cost analysis, is necessary.

\section{Strengths and Limitations}

The strengths of our study include that it was a prospective study, conducted at a single institution with a large and diverse inner city population. Although every effort was made to standardize collection methods, a potential limitation of our study includes provider differences in methods of vaginal and rectal GBS culture collection. On a similar note, a higher pregravid BMI amongst patients who were newly positive for GBS at delivery may have been a result of provider differences in specimen collection. In addition, a distinction was not made between intact and ruptured membranes upon the patient entering the study. Finally, 2 out of 11 patients who had a positive intrapartum GBS culture compared with their antepartum culture expired GBS status (over 5 weeks since collection) and had to be removed from analysis.

\section{Conclusion}

Our study showed that while there is evidence of agreement in GBS rectovaginal culture at 35 to 37 weeks with GBS colonization in labor, the level of agreement is weak. Moreover, the limited number of prior studies demonstrated varying levels of accuracy of the antepartum GBS culture in comparison to intrapartum results. Our study provides a recent perspective from a diverse, inner city population, and demonstrates that the 35- to 37-week GBS rectovaginal culture might be a poor predictor for GBS colonization in labor, leaving 11 (3.6\%) of fetuses vulnerable to infection and 33 (11\%) over treated. An essential aspect in the prevention of neonatal transmission of GBS is the correct identification of patients colonized with GBS intrapartum. This may indicate the utility of a rapid intrapartum screen to prevent inappropriate chemoprophylaxis. However, clinical significance of this difference in terms of neonatal outcomes warrants further study.

\section{Condensation}

Group beta Streptococcus (GBS) rectovaginal cultures at 35 to 37 weeks of gestation may be a poor predictor for GBS colonization in labor.

Note

This study was presented at the Society for Maternal-Fetal Medicine 38th annual Pregnancy Meeting, Dallas, TX, January 29 to February 3, 2018.

\section{Financial Supportl}

No financial support was obtained for this study.

Conflict of Interest

The authors report no conflicts of interest.

\section{References}

1 Centers for Disease Control and Prevention (CDC). Trends in perinatal group B streptococcal disease - United States, 20002006. MMWR Morb Mortal Wkly Rep 2009;58(05):109-112

2 Schrag SJ, Zywicki S, Farley MM, et al. Group B streptococcal disease in the era of intrapartum antibiotic prophylaxis. $\mathrm{N}$ Engl J Med 2000;342(01):15-20

3 Bromberger P, Lawrence JM, Braun D, Saunders B, Contreras R, Petitti DB. The influence of intrapartum antibiotics on the clinical spectrum of early-onset group B streptococcal infection in term infants. Pediatrics 2000;106(2, Pt. 1):244-250

4 Escobar GJ, Li DK, Armstrong MA, et al. Neonatal sepsis workups in infants $>/=2000$ grams at birth: A population-based study. Pediatrics 2000;106(2, Pt. 1):256-263

5 Pinto NM, Soskolne EI, Pearlman MD, Faix RG. Neonatal early-onset group B streptococcal disease in the era of intrapartum chemoprophylaxis: residual problems. J Perinatol 2003;23(04):265-271 
6 Pulver LS, Hopfenbeck MM, Young PC, et al. Continued early onset group B streptococcal infections in the era of intrapartum prophylaxis. J Perinatol 2009;29(01):20-25

7 Puopolo KM, Madoff LC, Eichenwald EC. Early-onset group B streptococcal disease in the era of maternal screening. Pediatrics 2005;115(05):1240-1246

8 Verani JR, McGee L, Schrag SJ; Division of Bacterial Diseases, National Center for Immunization and Respiratory Diseases, Centers for Disease Control and Prevention (CDC). Prevention of perinatal group B streptococcal disease-revised guidelines from CDC, 2010. MMWR Recomm Rep 2010;59(RR-10):1-36

9 American College of Obstetricians and Gynecologists Committee on Obstetric Practice. ACOG Committee Opinion No. 485: Prevention of early-onset group B streptococcal disease in newborns. Obstet Gynecol 2011;117(04):1019-1027

10 Boyer KM, Gadzala CA, Kelly PD, Burd LI, Gotoff SP. Selective intrapartum chemoprophylaxis of neonatal group B streptococcal early-onset disease. II. Predictive value of prenatal cultures. J Infect Dis 1983;148(05):802-809

11 Yancey MK, Schuchat A, Brown LK, Ventura VL, Markenson GR. The accuracy of late antenatal screening cultures in predicting genital group B streptococcal colonization at delivery. Obstet Gynecol 1996;88(05):811-815

12 Valkenburg-van den Berg AW, Houtman-Roelofsen RL, Oostvogel PM, Dekker FW, Dörr PJ, Sprij AJ. Timing of group B streptococcus screening in pregnancy: a systematic review. Gynecol Obstet Invest 2010;69(03):174-183

13 Rallu F, Barriga P, Scrivo C, Martel-Laferrière V, Laferrière C. Sensitivities of antigen detection and PCR assays greatly increased compared to that of the standard culture method for screening for group B streptococcus carriage in pregnant women. J Clin Microbiol 2006;44(03):725-728

14 Regan JA, Klebanoff MA, Nugent RP, et al; VIP Study Group. Colonization with group B streptococci in pregnancy and adverse outcome. Am J Obstet Gynecol 1996;174(04):1354-1360

15 Anthony BF, Eisenstadt R, Carter J, Kim KS, Hobel CJ. Genital and intestinal carriage of group B streptococci during pregnancy. J Infect Dis 1981;143(06):761-766
16 Dillon HC Jr, Gray E, Pass MA, Gray BM. Anorectal and vaginal carriage of group B streptococci during pregnancy. J Infect Dis 1982;145(06):794-799

17 McKenna DS, Matson S, Northern I. Maternal group B streptococcal (GBS) genital tract colonization at term in women who have asymptomatic GBS bacteriuria. Infect Dis Obstet Gynecol 2003;11 (04):203-207

18 Edwards RK, Clark P, Duff P. Intrapartum antibiotic prophylaxis 2: positive predictive value of antenatal group B streptococci cultures and antibiotic susceptibility of clinical isolates. Obstet Gynecol 2002;100(03):540-544

19 Kleweis SM, Cahill AG, Odibo AO, Tuuli MG. Maternal obesity and rectovaginal group B Streptococcus colonization at term. Infect Dis Obstet Gynecol 2015;2015:586767

20 Stapleton RD, Kahn JM, Evans LE, Critchlow CW, Gardella CM. Risk factors for group B streptococcal genitourinary tract colonization in pregnant women. Obstet Gynecol 2005;106(06): 1246-1252

21 Toyofuku M, Morozumi M, Hida M, et al. Effects of intrapartum antibiotic prophylaxis on neonatal acquisition of group B Streptococci. J Pediatr 2017;190:169-173.e1

22 Van Dyke MK, Phares CR, Lynfield R, et al. Evaluation of universal antenatal screening for group B streptococcus. N Engl J Med 2009; 360(25):2626-2636

23 Money D, Dobson S, Cole L, et al. An evaluation of a rapid real time polymerase chain reaction assay for detection of group B streptococcus as part of a neonatal group B streptococcus prevention strategy. J Obstet Gynaecol Can 2008;30(09): 770-775

24 Feuerschuette OHM, Silveira SK, Cancelier ACL, da Silva RM, Trevisol DJ, Pereira JR. Diagnostic yield of real-time polymerase chain reaction in the diagnosis of intrapartum maternal rectovaginal colonization by group B Streptococcus: a systematic review with meta-analysis. Diagn Microbiol Infect Dis 2018;91(02): 99-104 